\title{
Register Forschungsförderung der Deutschen Gesellschaft der Plastischen, Rekonstruktiven und Ästhetischen Chirurgen (DGPRÄC) und Forschungsförderungsbericht 2019/2020
}

\section{Registry Research Funding of the German Society of Plastic, Reconstructive and Aesthetic Surgeons (DGPRÄC) and Research Funding Report 2019/2020}

Autoren

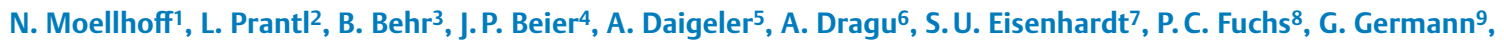
T. Hirsch ${ }^{10}$, R. E. Horch ${ }^{11}$, M. Infanger ${ }^{12}$, R. Jakubietz ${ }^{13}$, U. Kneser ${ }^{14,15}$, S. Langer ${ }^{16}$, M. Lehnhardt ${ }^{3}$, H. G. Machens ${ }^{17}$, P. Mailänder ${ }^{18}$, B. Reichert ${ }^{19}$, C. Radtke $^{20}$, D. J. Schaefer ${ }^{21}$, F. Siemers ${ }^{22}$, G. B. Stark7, H. U. Steinau ${ }^{23}$, P. M. Vogt ${ }^{24}$, R. E. Giunta ${ }^{1}$

Institute

1 Abteilung für Hand-, Plastische und Ästhetische Chirurgie, Klinikum der Universität München, LMU München

2 Hochschulzentrum für Plastische und Ästhetische, Hand- und Wiederherstellungschirurgie der Universität Regensburg

3 Klinik für Plastische Chirurgie und Schwerbrandverletzte, BG-Universitätsklinik Bergmannsheil

4 Klinik für Plastische Chirurgie, Hand- und Verbrennungschirurgie, Universitätsklinikum RWTH Aachen

5 Plastische Chirurgie, Hand-, Rekonstruktive und Verbrennungschirurgie an der Eberhard-Karls Universität/BC Unfallklinik Tübingen

6 Abteilung für Plastische und Handchirurgie, Universitätsklinikum Carl Gustav Carus, Dresden

7 Klinik für Plastische und Handchirurgie, Medizinische Fakultät, Universitätsklinikum Freiburg

8 Klinik für Plastische und Ästhetische Chirurgie, Handchirurgie - Schwerbrandverletztenzentrum, Klinikum Köln-Merheim

9 ETHIANUM, Klinik für Plastische, Ästhetische und Präventive Medizin am Universitätsklinikum Heidelberg

10 Plastische-, Rekonstruktive und Ästhetische Chirurgie, Fachklinik Hornheide, Plastische Chirurgie, Universitätsklinikum Münster

11 Plastisch- und Handchirurgische Klinik, Universitätsklinikum Erlangen, Friedrich-Alexander-Universität Erlangen-Nürnberg FAU

12 Klinik für Plastische, Wiederherstellungs- und Handchirurgie, Otto-von-Guericke-Universität, Magdeburg

13 Klinik für Unfall-, Hand-, Plastische und Wiederherstellungschirurgie/Sektion Plastische \& Ästhetische Chirurgie/ Universitätsklinikum Würzburg

14 Klinik für Hand-, Plastische \& Rekonstruktive Chirurgie/ Schwerbrandverletztenzentrum, BG-Unfallklinik Ludwigshafen

15 Klinik für Plastische und Handchirurgie, Ruprechts-KarlsUniversität Heidelberg
16 Abteilung für Plastische-, Ästhetische- und spezielle Handchirurgie, Universitätsklinikum Leipzig - AöR

17 Klinik und Poliklinik für Plastische Chirurgie und Handchirurgie/Klinikum rechts der Isar, Technische Universität München

18 Abteilung für Plastische Chirurgie, Handchirurgie, Zentrum für Schwerbrandverletzte, Universitätsklinikum Schleswig-Holstein, Campus Lübeck

19 Klinik für Plastische, Wiederherstellende und Handchirurgie, Zentrum für Schwerbrandverletzte, Klinikum Nürnberg-Süd, Universitätsklinik der Paracelsus Medizinischen Privatuniversität

20 Klinische Abteilung für Plastische und Rekonstruktive Chirurgie, Universitätsklinik für Chirurgie MedUni Wien

21 Plastische, Rekonstruktive, Ästhetische und Handchirurgie, Universitätsspital Basel

22 Klinik für Plastische Chirurgie und Handchirurgie, Brandverletztenzentrum, Berufsgenossenschaftliche Kliniken Bergmannstrost, Martin-Luther-Universität Halle-Wittenberg

23 Universitätsklinik für Unfallchirurgie/Orthopädie, Plastische und Rekonstruktive Chirurgie Schwerpunkt Sarkomchirurgie, WTZ

24 Klinik und Poliklinik für Plastische, Hand- und Wiederherstellungschirurgie, Medizinische Hochschule Hannover

Schlüsselwörter

Akademische Plastische Chirurgie, Forschungsförderung, DFG, BMBF

Key words

Academic Plastic Surgery, Research funding, DFG, BMBF

eingereicht 22.08.2020

akzeptiert $\quad 21.09 .2020$ 
Bibliografie

DOI https://doi.org/10.1055/a-1267-0388

Published online: 25.09.2020

Handchir Mikrochir Plast Chir 2021; 53: 110-118

(C) 2020. Thieme. All rights reserved.

Georg Thieme Verlag KG, Rüdigerstraße 14,

70469 Stuttgart, Germany

ISSN 0722-1819

Korrespondenzadresse

Univ.-Prof. Dr. med. Riccardo Giunta

Abteilung für Handchirurgie, Plastische Chirurgie

und Ästhetische Chirurgie

Klinikum der Universität München, LMU München

Pettenkoferstr. 8a, 80336 München

Germany

E-Mail: r.giunta@med.uni-muenchen.de

\section{ZUSAMMENFASSUNG}

Hintergrund Seit 2015/16 werden Forschungsanträge aus Sektionen, Abteilungen und Kliniken für Plastische Chirurgie an den Universitätskliniken Deutschlands durch die Deutsche Gesellschaft der Plastischen, Rekonstruktiven und Ästhetischen Chirurgen (DGPRÄC) gesammelt, ausgewertet und 2-jährlich veröffentlicht. Ziel ist es, dadurch die Forschungsleistung der Plastischen Chirurgen in Deutschland öffentlich sichtbar zu machen, um eine Stärkung der akademischen Forschungsstruktur in Deutschland zu erreichen.

Material und Methoden Die Leiter der universitären Einrichtungen wurden über den Verteiler der DGPRÄC kontaktiert und um Mitteilung der beantragten, in Begutachtung befindlichen, abgelehnten und geförderten Projekte jeglicher öffentlicher, nicht-öffentlicher, industrieller oder anderer Förderinstitute in die eigens dafür erstellte online Datenbank gebeten: https:// docs.google.com/forms/d/e/1FAIpQLSe6F5xmTyw-k7VKJx_2 jkPA4LBXsA0sgBGMrC3rx_4bHj6uzQ/viewform?usp=sf_link. Zusätzlich wurden Anträge über die öffentliche Datenbank der Deutschen Forschungsgemeinschaft (DFG), dem Geförderte Projekte Informationssystem (GEPRIS), identifiziert.

Ergebnisse Eine Gesamtzahl von 41 Förderanträgen aus den öffentlichen Förderinstituten DFG, BMBF, BMWi, BMG und EU konnte identifiziert werden. 75,6\% (31/41) der Anträge waren zum Zeitpunkt der Datenerhebung bereits bewilligt, davon waren 77,4\% (24/31) DFG-, 9,7\% (3/31) BMWi-, 6,5\% (2/31) EUund je 3,2\% (1/31) BMBF- und BMG-Anträge. Das durchschnittliche Fördervolumen entsprach 358301 Euro. Die bewilligten DFG-Anträge waren in 50,0\% (12/24) der Fälle ausschließlich bzw. unter anderem dem Fachkollegium 205-27 Orthopädie, Unfallchirurgie, rekonstruktive Chirurgie zugeordnet.

Schlussfolgerung Mithilfe der vorgelegten Forschungsförderungsberichte des Konvents der leitenden universitär tätigen Plastischen Chirurgen der DGPRÄC erfolgt die Veröffentlichung der exzellenten gemeinschaftlichen akademischen Forschungsleistung in unserem Fachgebiet.

\section{ABSTRACT}

Background Since 2015/16 the DGPRÄC collects, evaluates and publishes the research activities of academic sections, departments and clinics for plastic surgery at university hospitals in Germany, in order to raise the awareness of plastic surgical research performance.

Materials and Methods The directors of plastic surgical academic institutions were contacted via the DGPRÄC and asked to report any requested/approved and rejected research applications to public, non-public and industrial funding organizations. Data was collected in our previously established online database: https://docs.google.com/forms/d/e/1FAIpQLSe6F5xmTyw-k7VKJx_2jkPA4LBXsA0sgBGMrC3rx_4bHj6uzQ/viewform?usp=sf_link. In addition, applications were identified via the DFG's public database GEPRIS.

Results A total of 41 funding applications to the public funding institutes DFG, BMBF, BMWi, BMG and EU were identified. 75.6\% (31/41) of the applications had already been approved at the time of data collection, of which $77.4 \%$ (24/31) were DFG, $9.7 \%$ (3/31) were BMWi, 6.5\% (2/31) were EU and 3.2\% (1/31) were BMBF or BMG applications. The average funding amounted to 358301 Euro. In $50.0 \%$ (12/24) of the cases, the approved DFG proposals were assigned to the subject review board 205-27 Orthopedics, Trauma Surgery, Reconstructive Surgery.

Conclusion The continuous publication of plastic surgical research funding reports submitted by the convention of university plastic surgeons of the DGPRÄC portraits the excellent, collaborative research activity in the field of plastic surgery.

\section{Einleitung}

Die Forschungsförderungen durch nationale, öffentliche Forschungsinstitutionen (z. B. Förderungen der Deutschen Forschungsgemeinschaft [DFG], des Bundesministeriums für Bildung und Forschung [BMBF], des Bundesministeriums für Wirtschaft und Energie [BMWi] oder des Bundesministeriums für Gesundheit [BMG]) spielen in der universitären Medizin eine große Rolle. Insbesondere für Fachgebiete mit meist noch zu wenig universitärer Infrastruktur, wie der Plastischen Chirurgie, ist das Einwerben von Forschungsmitteln ein wichtiger Maßstab, um beispielsweise von einer Sektion zur eigenständigen Abteilung oder Klinik zu wach- sen [1-4]. Gleichzeitig kann das Einwerben großer Forschungsförderungen das Fachgebiet der Plastischen Chirurgie gegenüber den großen, traditionellen chirurgischen Kliniken wissenschaftlich konkurrenzfähiger machen.

Seit 2015 werden die Forschungsanträge aus Sektionen, Abteilungen und Kliniken für Plastische Chirurgie an den Universitätskliniken Deutschlands durch die Deutsche Gesellschaft der Plastischen, Rekonstruktiven und Ästhetischen Chirurgen (DGPRÄC) gesammelt und im Rahmen 2-jährlicher Forschungsberichte veröffentlicht $[5,6]$. 
Während die Plastische Chirurgie bedauerlicherweise noch immer nicht als eigenständiges Fachgebiet in der Fächerstruktur der DFG abgebildet ist, wurde mittlerweile zumindest das Fachkollegium 205-27 um den Begriff „rekonstruktive Chirurgie“ erweitert. Dieser entspricht im Gegensatz zu den anderen beiden Begriffen, die heute das chirurgische Fachgebiet „Orthopädie und Unfallchirurgie“ zusammenfassen, keinem Fachgebiet und wird von verschiedenen chirurgischen Fachgebieten beansprucht. Dennoch ist dieser Wandel als kleiner Schritt in die Richtung der wirklichen Repräsentanz in der DFG des seit 1993 existierenden Fachgebiets „Plastische Chirurgie“ zu werten. Erfreulicherweise repräsentiert nun immerhin auch ein hochrangiger Wissenschaftler aus der Plastischen Chirurgie gemeinsam mit zweien aus dem Bereich Orthopädie und Unfallchirurgie das DFG-Fachkollegium Orthopädie, Unfallchirurgie, rekonstruktive Chirurgie mit.

Ziel der Studie ist die Stärkung der akademischen plastisch-chirurgischen Forschungsstruktur in Deutschland. Aus diesem Grund wurden nur Anträge der universitären Einrichtungen in Deutschland berücksichtigt. Gleichzeitig soll mittelfristig die Einrichtung eines eigenständigen Fachkollegiums „Plastische, Rekonstruktive und Ästhetische Chirurgie“, z. B. bei der DFG, angestrebt werden, um eine fachgerechte Begutachtung analog zu den anderen Fachgebieten der Chirurgie sicherzustellen. Die vorliegende Studie dient daher dazu, die umfangreichen Forschungsaktivitäten, die derzeit oft unter anderen chirurgischen Fachgebieten subsumiert werden und daher nicht als Gesamtheit für das Fachgebiet Plastische Chirurgie wahrgenommen werden, sichtbar zu machen und die hohe qualitative Produktivität und Innovation in der Plastischen Chirurgie darzustellen.

Im Folgenden wird die Forschungsleistung der universitären Plastischen Chirurgen in Deutschland für den Zeitraum 2019/2020 dargestellt.

\section{Methoden}

Äquivalent zum Vorgehen in den vorherigen Forschungsförderungsberichten 2015/2016 [6] und 2017/2018 [5] wurden Forschungsanträge universitärer Sektionen/Abteilungen und Kliniken für Plastische Chirurgie in Deutschland bei öffentlichen Förderinstitutionen für den Zeitraum 2019 und/oder 2020 gesammelt und ausgewertet. Dazu wurde einerseits die öffentliche Datenbank der DFG, das Geförderte Projekte Informationssystem (GEPRIS), verwendet. [7] Zusätzlich wurden die Leiter der jeweiligen universitären Einrichtungen über den Verteiler der DGPRÄC kontaktiert und um Mitteilung der beantragten, in Begutachtung befindlichen, abgelehnten und geförderten Projekte jeglicher öffentlicher, nichtöffentlicher, industrieller oder anderer Förderinstitute gebeten. Diese wurden in der eigens erstellten online Datenbank gesammelt: https://docs.google.com/forms/d/e/1FAlpQLSe6F5xmTyw-k7VKJ x_2jkPA4LBXsA0sgBGMrC3rx_4bHj6uzQ/viewform?usp=sf_link

Anträge der großen öffentlichen Förderinstitute (DFG, BMBF, BMWi, BMG, EU) wurden zur verbesserten Übersicht gesondert dargestellt ( $\vee$ Tab. 1) und getrennt von Anträgen anderer öffentlicher, nicht-öffentlicher und industrieller Förderinstitute ( $>$ Tab. 2 ) ausgewertet.

\section{Ergebnisse}

Im Untersuchungszeitraum 2019/2020 wurden an 10 universitären plastisch-chirurgischen Standorten Fördermittel von DFG, BMBF, BMWi, BMG oder EU beantragt. Dabei gingen von einer Institution zwischen 1 und 13 Anträge aus.

Eine Gesamtzahl von 41 Förderanträgen aus den öffentlichen Förderinstituten DFG, BMBF, BMWi, BMG und EU konnte nach Auswertung der Online-Datenbank und des GEPRIS identifiziert werden. Davon waren 73,2\% (30/41) DFG-, 9,8\% (4/41) BMBF-, je 7,3\% (3/41) BMWi- und EU- und 2,4\% (1/41) BMG-Anträge.

75,6\% (31/41) der Anträge waren zum Zeitpunkt der Datenerhebung bereits bewilligt, davon waren 77,4\% (24/31) DFG-, 9,7\% (3/31) BMWi-, 6,5\% (2/31) EU- und je 3,2\% (1/31) BMBF- und BMGAnträge.

9,8\% (4/41) aller Anträge befanden sich während der Auswertung noch in Begutachtung. Davon waren 75,0\% (3/4) bei der DFG und 25,0\% (1/4) beim BMBF eingereicht. 14,6\% (6/41) aller Anträge an öffentlichen Förderinstituten wurden abgelehnt. In 50,0\% (3/6) der Fälle waren diese bei der DFG, in 33,3\% (2/6) der Fälle beim BMBF und in 16,7 \% (1/6) der Fälle als EU-Förderung beantragt worden.

Die bewilligten DFG-Anträge waren in 50,0\% (12/24) der Fälle ausschließlich bzw. unter anderem dem Fachkollegium 205-27 Orthopädie, Unfallchirurgie, rekonstruktive Chirurgie zugeordnet. In 29,2 \% (7/24) der Fälle waren die Anträge ausschließlich bzw. unter anderem dem Fachkollegium Allgemein- u. Viszeralchirurgie zugeordnet. In 33,3\% (8/24) der Fälle waren die Anträge ausschließlich bzw. unter anderem sonstigen Fachkollegien wie z. B. Biomaterialien, Biophysik oder Zellbiologie zugeordnet.

In 37,5\% (9/24) der Fälle wurden die DFG-Anträge als Sachbeihilfe eingestuft. Je 16,7\% (4/24) der DFG-Anträge waren Großgeräteanträge, Forschungsstipendien oder Transregios. 8,3\% der Fälle (2/24) waren Heisenberg-Professuren und 4,2 \% (1/24) der Fälle waren Schwerpunkprogrammen zugeordnet.

In 67,7\% (21/31) der bewilligten Anträge öffentlicher Förderinstitute wurde die Fördersumme durch die befragten Chirurgen angegeben. Diese betrug insgesamt 7524326 Euro, was einem durchschnittlichen Fördervolumen von 358301 Euro entspricht.

Neben den Anträgen bei oben genannten öffentlichen Förderinstitutionen (DFG, BMBF, BMWi, BMG und EU) sind der Vollständigkeit halber, wie auch im vorangegangenen Forschungsbericht 2017/2018 [5], in > Tab. 2 auf der Internetplattform angegebene Forschungsanträge anderer öffentlicher, nicht-öffentlicher oder industrieller Institute dargestellt. Neben den oben genannten Anträgen von DFG, BMBF, BMWi, BMG und EU wurden 52 sonstige Anträge angegeben. Von diesen waren 84,6\% (44/52) bewilligt. Das genaue Fördervolumen der bewilligten Anträge wurde in 97,7\% (43/44) der bewilligten Fälle angegeben. Dieses betrug insgesamt 6025645 Euro, was einem durchschnittlichen Fördervolumen von 143468 Euro entspricht.

\section{Diskussion}

Im internationalen Vergleich zeigt sich, dass nicht-chirurgische Fächer im Gegensatz zu chirurgischen Fächern deutlich mehr Forschungsanträge bei nationalen und öffentlichen Forschungsinstituten einholen. Daten aus den USA legen dar, dass die Finanzierung 
> Tab. 1 Übersicht über die bewilligte Forschungsförderung großer öffentlicher Fördereinrichtungen (DFG, BMBF, BMWi, BMG und EU) nach Standorten.

\begin{tabular}{|c|c|c|c|c|c|}
\hline Uniklinik & $\begin{array}{l}\text { Förder- } \\
\text { institut } \\
\text { und Art der } \\
\text { Förderung }\end{array}$ & Projekttitel & Zuordnung & Zeitraum & $\begin{array}{l}\text { Förder- } \\
\text { summe } \\
\text { (Euro) }\end{array}$ \\
\hline Aachen & $\begin{array}{l}\text { DFG } \\
\text { Sachbeihilfe }\end{array}$ & $\begin{array}{l}\text { Die Rolle der Makrophagen migrations- } \\
\text { inhibierenden Faktor (MIF)-Proteinfamilie in } \\
\text { Wundheilung und Übergewicht }\end{array}$ & Allgemein- u. Viszeralchirurgie & $2018-2021$ & 340000 \\
\hline Aachen & $\begin{array}{l}\text { EU, Horizon } \\
2020\end{array}$ & PeriGo! & k. A. & 2019-2020 & 150000 \\
\hline Aachen & $\begin{array}{l}\text { EFRE-NRW, } \\
\text { Europäische } \\
\text { Fonds für } \\
\text { regionale } \\
\text { Entwicklung }\end{array}$ & Fibrograft & k. A. & 2020-2022 & 620000 \\
\hline Bochum & $\begin{array}{l}\text { DFG Sach- } \\
\text { beilhilfe }\end{array}$ & $\begin{array}{l}\text { Interventionen im Sphingosin-1 Phosphat Signal- } \\
\text { weg zur Verbesserung der Knochenregeneration } \\
\text { nach posttraumatischer Osteomyelitis }\end{array}$ & $\begin{array}{l}\text { Orthopädie, Unfallchirurgie, } \\
\text { rekonstruktive Chirurgie }\end{array}$ & $2020-2022$ & 237700 \\
\hline Bochum & $\begin{array}{l}\text { DFG For- } \\
\text { schungs- } \\
\text { stipendium }\end{array}$ & $\begin{array}{l}\text { Osteoskeletales Regenerationspotential von } \\
\text { Exosomen von Stammzellen aus Fettgewebe } \\
\text { unter Inhibition des Transforming growth factor } \\
\beta \text {-Signalweges }\end{array}$ & Allgemein- und Viszeralchirurgie & seit 2019 & k. A. \\
\hline Bochum & $\begin{array}{l}\text { DFG Schwer- } \\
\text { punkt- } \\
\text { programm }\end{array}$ & $\begin{array}{l}\text { BURNTwo - Diagnose von Brandwunden mit mikro- } \\
\text { wellenbasierter Nahfeldbildgebung - Phase II }\end{array}$ & $\begin{array}{l}\text { Nachrichten- und Hochfrequenz- } \\
\text { technik, Kommunikationstechnik und } \\
\text {-netze, theoretische Elektrotechnik, } \\
\text { Orthopädie, Unfallchirurgie, rekon- } \\
\text { struktive Chirurgie }\end{array}$ & seit 2015 & k. A. \\
\hline Bochum & $\begin{array}{l}\text { DFG Sach- } \\
\text { beilhilfe }\end{array}$ & $\begin{array}{l}\text { Mesenchymale Stammzellen und Wnt-Proteine in } \\
\text { der Therapie der posttraumatischen Osteomyelitis }\end{array}$ & $\begin{array}{l}\text { Orthopädie, Unfallchirurgie, } \\
\text { rekonstruktive Chirurgie }\end{array}$ & $2017-2020$ & 191400 \\
\hline Bochum & BMWi & $\begin{array}{l}\text { Entwicklung einer Wundauflage für austherapierte } \\
\text { chronische Wunden mit integriertem, aus Lucilia } \\
\text { sericata gewonnenem Madenextrakt }\end{array}$ & k. A. & $2018-2020$ & 190000 \\
\hline Bochum & BMWi & $\begin{array}{l}\text { Entwicklung einer intramuskulären Elektro- } \\
\text { stimulations-Therapie für Schwerbrandverletzte“ }\end{array}$ & k. A. & $2020-2022$ & 190000 \\
\hline Erlangen & $\begin{array}{l}\text { DFG } \\
\text { Transregios }\end{array}$ & $\begin{array}{l}\text { Biofabrikation zellularisierter und im AV Loop } \\
\text { vaskularisierter Gewebecontainer für die } \\
\text { Transplantation wirkstoffproduzierender Zellen }\end{array}$ & $\begin{array}{l}\text { Hämatologie, Onkologie, Transfusions- } \\
\text { medizin, Biomaterialien; Verbund- } \\
\text { projekt mit Teilprojekt der Plastischen } \\
\text { Chirurgie }\end{array}$ & seit 2018 & k. A. \\
\hline Erlangen & $\begin{array}{l}\text { DFG } \\
\text { Transregios }\end{array}$ & $\begin{array}{l}\text { Analyse von Tumor Dormancy und Progression } \\
\text { in biofabrizierten und in vivo vaskularisierten 3D } \\
\text { Modellen }\end{array}$ & $\begin{array}{l}\text { Zellbiologie, Biomaterialien; Verbund- } \\
\text { projekt mit Teilprojekt der Plastischen } \\
\text { Chirurgie }\end{array}$ & seit 2018 & k. A. \\
\hline Erlangen & $\begin{array}{l}\text { DFG } \\
\text { Sachbeihilfe }\end{array}$ & $\begin{array}{l}\text { Vaskularisation von neuromuskulären Konstrukten } \\
\text { auf PCL-Kollagen I-PEO-Nanofaserscaffolds }\end{array}$ & $\begin{array}{l}\text { Orthopädie, Unfallchirurgie, } \\
\text { rekonstruktive Chirurgie }\end{array}$ & seit 2019 & 169348 \\
\hline Freiburg & $\begin{array}{l}\text { DFG } \\
\text { Heisenberg } \\
\text { Professur }\end{array}$ & $\begin{array}{l}\text { Die Rolle der angeborenen Immunantwort in } \\
\text { Entzündungsreaktionen und entzündlichen } \\
\text { Erkrankungen: Erforschung und Entwicklung neuer } \\
\text { therapeutischer Ansätze }\end{array}$ & Allgemein-u. Viszeralchirurgie & 2020-2022 & 200000 \\
\hline Freiburg & $\begin{array}{l}\text { DFG } \\
\text { Sachbeihilfe }\end{array}$ & $\begin{array}{l}\text { Regulation der angeborenen Immunantwort in der } \\
\text { vascularized composite allotransplantation (VCA) } \\
\text { durch C-reaktives Protein }\end{array}$ & Allgemein-u. Viszeralchirurgie & $2020-2023$ & 460000 \\
\hline Freiburg & $\begin{array}{l}\text { DFG } \\
\text { Großgeräte }\end{array}$ & $\begin{array}{l}\text { Multiphotonen Laser Scanning Mikroskop für die } \\
\text { intravitale Bildgebung }\end{array}$ & Medizin Allgemein & $2020-2023$ & 700000 \\
\hline Freiburg & $\begin{array}{l}\text { DFG } \\
\text { Heisenberg } \\
\text { Professur }\end{array}$ & $\begin{array}{l}\text { Die Rolle der angeborenen Immunantwort in } \\
\text { Entzündungsreaktionen und entzündlichen } \\
\text { Erkrankungen: Erforschung und Entwicklung neuer } \\
\text { therapeutischer Ansätze }\end{array}$ & Allgemein-u. Viszeralchirurgie & $2017-2020$ & 300000 \\
\hline
\end{tabular}


- Tab. 1 Fortsetzung.

\begin{tabular}{|c|c|c|c|c|c|}
\hline Uniklinik & $\begin{array}{l}\text { Förder- } \\
\text { institut } \\
\text { und Art der } \\
\text { Förderung }\end{array}$ & Projekttitel & Zuordnung & Zeitraum & $\begin{array}{l}\text { Förder- } \\
\text { summe } \\
\text { (Euro) }\end{array}$ \\
\hline $\begin{array}{l}\text { Freiburg; } \\
\text { LMU } \\
\text { München }\end{array}$ & $\begin{array}{l}\text { DFG } \\
\text { Sachbeihilfe }\end{array}$ & $\begin{array}{l}\text { Zirkulierende Tumor DNA als nicht-invasiver } \\
\text { diagnostischer Biomarker bei Weichteilsarkomen }\end{array}$ & $\begin{array}{l}\text { Orthopädie, Unfallchirurgie, } \\
\text { rekonstruktive Chirurgie; } \\
\text { Allgemein- u. Viszeralchirurgie }\end{array}$ & $2018-2021$ & 330000 \\
\hline Freiburg & $\begin{array}{l}\text { DFG } \\
\text { Sachbeihilfe }\end{array}$ & $\begin{array}{l}\text { Die Interaktion von C-reaktivem Protein (CRP) } \\
\text { mit dem Komplementsystem in der Pathogenese, } \\
\text { Diagnostik, und Therapie der posttraumatischen } \\
\text { Entzündungsantwort }\end{array}$ & $\begin{array}{l}\text { Orthopädie, Unfallchirurgie, } \\
\text { rekonstruktive Chirurgie }\end{array}$ & $2017-2020$ & 200000 \\
\hline Freiburg & $\begin{array}{l}\text { DFG } \\
\text { Sachbeihilfe }\end{array}$ & $\begin{array}{l}\text { C-reaktives Protein (CRP) als pathogenetischer } \\
\text { Faktor bei Entzündung und Ischämie/Reperfusion: } \\
\text { Therapeutische Implikationen }\end{array}$ & Allgemein-u. Viszeralchirurgie & $2016-2020$ & 500000 \\
\hline Freiburg & $\begin{array}{l}\text { DFG } \\
\text { Sachbeihilfe }\end{array}$ & $\begin{array}{l}\text { 4D-Bioprinting von vaskularisiertem Knochenge- } \\
\text { webe und Evaluation der Blutgefäß- und Knochen- } \\
\text { bildung in einem orthotopen Knochendefektmodell }\end{array}$ & $\begin{array}{l}\text { Orthopädie, Unfallchirurgie, } \\
\text { rekonstruktive Chirurgie, } \\
\text { Bioverfahrenstechnik }\end{array}$ & seit 2015 & k. A. \\
\hline Freiburg & $\begin{array}{l}\text { BMBF } \\
\text { Forschungs- } \\
\text { verbund }\end{array}$ & $\begin{array}{l}\text { Entwicklung und Erforschung einer Plattform } \\
\text { für das 3D-Bio-Printing von künstlichem Gewebe } \\
\text { in einem Netzwerk kleiner und mittlerer Unterneh- } \\
\text { men (3D-Bio-Net) }\end{array}$ & k. A. & 2020 & 243326 \\
\hline Heidelberg & $\begin{array}{l}\text { DFG For- } \\
\text { schungs- } \\
\text { stipendium }\end{array}$ & $\begin{array}{l}\text { Korneale Reinnervation in der Therapie der } \\
\text { Neurotrophen Keratopathie - Evaluation topischer } \\
\text { und mikrochirurgischer Therapieansätze in } \\
\text { einem standardisierten, präklinischen Langzeit- } \\
\text { modell der kornealen Denervation in transgenen } \\
\text { Thy1-GFP + Ratten }\end{array}$ & $\begin{array}{l}\text { Augenheilkunde, klinische Neurologie; } \\
\text { Neurochirurgie und Neuroradiologie }\end{array}$ & seit 2019 & k. A. \\
\hline $\begin{array}{l}\text { Heidelberg/ } \\
\text { Ludwigshafen }\end{array}$ & $\begin{array}{l}\text { DFG For- } \\
\text { schungs- } \\
\text { stipendium }\end{array}$ & $\begin{array}{l}\text { Die Rolle des Tumorsuppressorgens NDRG2 in } \\
\text { Angiogenese und Wundheilung }\end{array}$ & $\begin{array}{l}\text { Orthopädie, Unfallchirurgie, } \\
\text { rekonstruktive Chirurgie }\end{array}$ & seit 2018 & k. A. \\
\hline $\begin{array}{l}\text { Heidelberg/ } \\
\text { Ludwigshafen }\end{array}$ & $\begin{array}{l}\text { DFG For- } \\
\text { schungs- } \\
\text { stipendium }\end{array}$ & $\begin{array}{l}\text { Funktionelle Ergebnisse und Reinnervationstendez } \\
\text { von vaskularisierten Komposit-Auto und -allotrans- } \\
\text { plantationen nach ex-vivo Perfusion des Hinterlaufs } \\
\text { der Ratte zur Reduktion des Ischämie-Reperfusions- } \\
\text { schaden }\end{array}$ & $\begin{array}{l}\text { Orthopädie, Unfallchirurgie, } \\
\text { rekonstruktive Chirurgie }\end{array}$ & seit 2020 & k. A. \\
\hline $\begin{array}{l}\text { LMU } \\
\text { München }\end{array}$ & $\begin{array}{l}\text { DFG } \\
\text { Großgeräte }\end{array}$ & $\begin{array}{l}\text { Einsatz von Floureszenzmikroskop in der Mikro- } \\
\text { chirurgie }\end{array}$ & $\begin{array}{l}\text { Orthopädie, Unfallchirurgie, } \\
\text { rekonstruktive Chirurgie }\end{array}$ & $2019-2025$ & 409000 \\
\hline $\begin{array}{l}\text { LMU } \\
\text { München }\end{array}$ & $\begin{array}{l}\text { DFG } \\
\text { Großgeräte }\end{array}$ & 3D Imaging System & $\begin{array}{l}\text { Orthopädie, Unfallchirurgie, } \\
\text { rekonstruktive Chirurgie }\end{array}$ & 2019-2022 & 277200 \\
\hline Magdeburg & DLR/BMWi & $\begin{array}{l}\text { Effekte der Schwerelosigkeit auf Gefäß,- und } \\
\text { Sphäroidbildung humaner Zellen sowie auf Wund- } \\
\text { heilung und Herzmorphologie }\end{array}$ & $\begin{array}{l}\text { Mikrogravitation und Translationale } \\
\text { Regenerative Medizin }\end{array}$ & 2019-2022 & 685752 \\
\hline Regensburg & $\begin{array}{l}\text { DFG } \\
\text { Großgeräte }\end{array}$ & Mikroskop Kinevo & $\begin{array}{l}\text { Orthopädie, Unfallchirurgie, rekon- } \\
\text { struktive Chirurgie }\end{array}$ & 2019 & 685000 \\
\hline TU München & $\begin{array}{l}\text { Bundes- } \\
\text { ministerium } \\
\text { für Gesund- } \\
\text { heit - For- } \\
\text { schergruppe }\end{array}$ & Künstliche Intelligenz für Prophylaxe von Dekubitus & Plastische Chirurgie & $2020-2022$ & 445600 \\
\hline Würzburg & $\begin{array}{l}\text { DFG } \\
\text { Transregios }\end{array}$ & $\begin{array}{l}\text { Biofabrikation eines 3D Modells zur funktionalen } \\
\text { Untersuchung stromaler Einflussfaktoren auf das } \\
\text { Verhalten von Brustkrebszellen }\end{array}$ & $\begin{array}{l}\text { Biophysik, Biomaterialien, Verbund- } \\
\text { projekt mit Teilprojekt der Plastischen } \\
\text { Chirurgie }\end{array}$ & seit 2018 & k. A. \\
\hline Würzburg & $\begin{array}{l}\text { DFG } \\
\text { Transregios }\end{array}$ & $\begin{array}{l}\text { Hyaluronsäure-basierte Hydrogelplattform mit } \\
\text { multifunktionalen Vernetzern zur kontrollierten } \\
\text { Differenzierung mesenchymaler Stammzellen (A02) }\end{array}$ & $\begin{array}{l}\text { Biomaterialien, Verbundprojekt mit } \\
\text { Teilprojekt der Plastischen Chirurgie }\end{array}$ & seit 2018 & k. A. \\
\hline
\end{tabular}


> Tab.2 Übersicht über die bewilligte Forschungsförderung öffentlicher, nicht-öffentlicher, industrieller oder anderer Institute (exklusive DFG, BMBF, BMWi, BMG und EU) nach Standorten.

\begin{tabular}{|c|c|c|c|c|c|}
\hline Uniklinik & $\begin{array}{l}\text { Förderinstitut und Art der } \\
\text { Förderung }\end{array}$ & Projekttitel & Zuordnung & Zeitraum & $\begin{array}{l}\text { Förder- } \\
\text { summe } \\
\text { (Euro) }\end{array}$ \\
\hline Aachen & VW-Stiftung & $\begin{array}{l}\text { Why is There No Antimicrobial Sensitivity Testing } \\
\text { Available for Antiseptics? }\end{array}$ & k. A. & 2018-2019 & 100000 \\
\hline Aachen & $\begin{array}{l}\text { START - Forschungsförderungs- } \\
\text { programm der Medizinischen } \\
\text { Fakultät der RWTH Aachen }\end{array}$ & $\begin{array}{l}\text { 3D-Bioprinting von Skelettmuskelgewebe aus } \\
\text { humanen ASC auf textilen Gerüststrukturen }\end{array}$ & k. A. & 2019-2020 & 130000 \\
\hline Aachen & $\begin{array}{l}\text { START - Forschungsförderungs- } \\
\text { programm der Medizinischen } \\
\text { Fakultät der RWTH Aachen }\end{array}$ & $\begin{array}{l}\text { Bioengineering von Lymphknotengewebe zur } \\
\text { Therapie von Lymphödemen }\end{array}$ & k. A. & 2019-2021 & 25000 \\
\hline Aachen & $\begin{array}{l}\text { START - Forschungsförderungs- } \\
\text { programm der Medizinischen } \\
\text { Fakultät der RWTH Aachen }\end{array}$ & $\begin{array}{l}\text { Die Rolle des Endocannabinoiden Systems der } \\
\text { mesenchymalen Stammzellen aus dem Fett- } \\
\text { gewebe bei der Geweberegeneration, Wund- } \\
\text { heilungsprozessen und Entzündungsreaktionen }\end{array}$ & k. A. & 2019-2020 & 20000 \\
\hline Aachen & IZKF & $\begin{array}{l}\text { ASC-based in vitro and in vivo bone tissue } \\
\text { engineering for bone defect reconstruction }\end{array}$ & k. A. & $2020-2023$ & 134000 \\
\hline Bochum & DGUV & $\begin{array}{l}\text { Charakterisierung der systemischen Kinetik von } \\
\text { Effektormolekülen der epithelialen Immunantwort } \\
\text { und mikroRNA bei schweren Brandverletzungen } \\
\text { und Sepsis }\end{array}$ & k. A. & 2018-2020 & 279000 \\
\hline Bochum & FoRUM & Rotationsstelle & k. A. & 2019 & 67000 \\
\hline Bochum & FoRUM & Rotationsstelle & k. A. & 2020 & 67000 \\
\hline Bochum & FoRUM & Genexpression im diabetischen Knochen & k. A. & $2018-2020$ & 43500 \\
\hline Bochum & FoRUM & Diabetische Neuropathie & k. A. & $2018-2020$ & 37500 \\
\hline Bochum & FoRUM & $\begin{array}{l}\text { Myostatin im Ischämie-Reperfusionsschaden des } \\
\text { Muskels }\end{array}$ & k. A. & 2020-2021 & 57000 \\
\hline Erlangen & ELAN-Fonds Uni Erlangen & $\begin{array}{l}\text { The effect of anti-tumor therapy on the autotaxin- } \\
\text { LPA axis in breast cancer }\end{array}$ & k. A. & 2019-2021 & 49825 \\
\hline Erlangen & ELAN-Fonds Uni Erlangen & $\begin{array}{l}\text { Vascularization and bone formation of cell-loaded } \\
\text { hydrogel matrices in the rat AV loop model }\end{array}$ & k. A. & 2019-2021 & 49146 \\
\hline Erlangen & $\begin{array}{l}\text { ELAN-Fonds Uni Erlangen - } \\
\text { Forschergruppe }\end{array}$ & Intravitalmikroskopie im AV-Loop & $\begin{array}{l}\text { Plastische } \\
\text { Chirurgie }\end{array}$ & 2019 & 49370 \\
\hline Freiburg & MWK - Personalstelle & Regenerative Ansätze in der Plastischen Chirurgie & $\begin{array}{l}\text { Plastische } \\
\text { Chirurgie }\end{array}$ & 2019-2020 & $\begin{array}{l}75 \% \text { einer } \\
\text { Vollzeit } \\
\text { Personal- } \\
\text { stelle }\end{array}$ \\
\hline Freiburg & $\begin{array}{l}\text { Dr. Robert Pfleger-Stiftung } \\
\text { Sachbeihilfe }\end{array}$ & $\begin{array}{l}\text { Modulation der Immunogenität und des } \\
\text { regenerativen Potenzials fötaler endothelialer } \\
\text { Progenitorzellen durch CRISPR/Cas9-vermittelte } \\
\text { Inaktivierung der MHC Expression. }\end{array}$ & k. A. & 2020 & 61628 \\
\hline Hannover & $\begin{array}{l}\text { Fritz-Behrens-Stiftung } \\
\text { Sachbeihilfe }\end{array}$ & $\begin{array}{l}\text { Verwendung von nativer Spinnenseide zur } \\
\text { Schaffung einer optimalen Mikroumgebung für die } \\
\text { Regeneration }\end{array}$ & k. A. & $2016-2020$ & 80000 \\
\hline Hannover & $\begin{array}{l}\text { Klaus Tschira Stiftung } \\
\text { Sachbeihilfe }\end{array}$ & $\begin{array}{l}\text { Amphibien und Spinnen begeistern Schüler*innen } \\
\text { für die Wissenschaft }\end{array}$ & $\begin{array}{l}\text { MINT-Fächer, } \\
\text { Pädagogik }\end{array}$ & $2019-2020$ & 31000 \\
\hline Hannover & MHH Präsidium Sachbeihilfe & Etablierung der PÄHW-Hautbank & k. A. & 2017-2019 & 300000 \\
\hline Hannover & Industrie & SCAMAG Studie & Handchirurgie & $2019-2020$ & 750000 \\
\hline Hannover & VHV-Stiftung Sachbeihilfe & $\begin{array}{l}\text { Entwicklung eines Biopharmazeutikums zur } \\
\text { lokalen Immunsuppression von Haut }\end{array}$ & k. A. & 2020 & 90000 \\
\hline Hannover & E. Braun Stiftung Sachbeihilfe & Einfluss von wIRA auf Meshgrafts & k. A. & 2020-2021 & 70000 \\
\hline Hannover & $\begin{array}{l}\text { MHHplus Förderstiftung } \\
\text { Sachbeihilfe }\end{array}$ & Regenerationsforschung & k. A. & 2020 & 35000 \\
\hline Hannover & $\begin{array}{l}\text { Rotary Club Leineschlösschen } \\
\text { Sachbeihilfe }\end{array}$ & Arterhaltende Maßnahmen für Querzahnmolche 1 & k. A. & 2019-2020 & 4000 \\
\hline Hannover & $\begin{array}{l}\text { MHHplus Förderstiftung } \\
\text { Sachbeihilfe }\end{array}$ & Arterhaltende Maßnahmen für Querzahnmolche 2 & k. A. & 2019-2020 & 500 \\
\hline
\end{tabular}


$>$ Tab. 2 Fortsetzung.

\begin{tabular}{|c|c|c|c|c|c|}
\hline Uniklinik & $\begin{array}{l}\text { Förderinstitut und Art der } \\
\text { Förderung }\end{array}$ & Projekttitel & Zuordnung & Zeitraum & $\begin{array}{l}\text { Förder- } \\
\text { summe } \\
\text { (Euro) }\end{array}$ \\
\hline Hannover & $\begin{array}{l}\text { Gesellschaft der Freunde der } \\
\text { Medizinischen Hochschule } \\
\text { Hannover e. V. Sachbeihilfe }\end{array}$ & $\begin{array}{l}\text { Evaluation gentechnischer Strategien zur } \\
\text { Entwicklung universeller Spenderhaut }\end{array}$ & k. A. & 2019-2020 & 1000 \\
\hline $\begin{array}{l}\text { Heidelberg/ } \\
\text { Ludwigshafen }\end{array}$ & Private Stiftung - Sachbeihilfe & $\begin{array}{l}\text { Etablierung eines Xenograft Modells venöser } \\
\text { Malformationen }\end{array}$ & $\begin{array}{l}\text { Plastische } \\
\text { Chirurgie }\end{array}$ & 2024 & 35000 \\
\hline $\begin{array}{l}\text { Heidelberg/ } \\
\text { Ludwigshafen }\end{array}$ & DGUV - Forschergruppe & $\begin{array}{l}\text { Evaluation des ICF-Rehabilitationskonzepts } \\
\text { thermische Verletzungen }\end{array}$ & $\begin{array}{l}\text { Plastische } \\
\text { Chirurgie }\end{array}$ & 2019-2020 & 510187 \\
\hline $\begin{array}{l}\text { Heidelberg/ } \\
\text { Ludwigshafen }\end{array}$ & DGUV - Forschergruppe & $\begin{array}{l}\text { Optimierte Primär- und Verlaufsdiagnostik von } \\
\text { peripheren Nervenläsionen }\end{array}$ & Handchirurgie & $2019-2020$ & 188453 \\
\hline $\begin{array}{l}\text { Heidelberg/ } \\
\text { Ludwigshafen }\end{array}$ & Rolf Schwiete Stiftung & Kardiale Langzeitfolgen schwerer Verbrennungen & $\begin{array}{l}\text { Verbrennungsme- } \\
\text { dizin, Kardiologie, } \\
\text { Immunologie }\end{array}$ & 2019-2022 & 240000 \\
\hline $\begin{array}{l}\text { LMU } \\
\text { München }\end{array}$ & Eigenfetttransplantation & $\begin{array}{l}\text { Eigenfetttransplantation zur Behandlung von Rhiz- } \\
\text { arthrose - Analyse regenerativer Mechanismen }\end{array}$ & $\begin{array}{l}\text { Hand- und Plas- } \\
\text { tische Chirurgie }\end{array}$ & $2019-2020$ & 50939 \\
\hline $\begin{array}{l}\text { LMU } \\
\text { München }\end{array}$ & $\begin{array}{l}\text { Antrag an den Verein zur } \\
\text { Förderung von Wissenschaft } \\
\text { und Forschung LMU München }\end{array}$ & $\begin{array}{l}\text { Das Sekretom von adipose-derived stem cells } \\
\text { (ADSCs) als Therapieansatz für chronisch ent- } \\
\text { zündliche Erkrankung am Beispiel von Arthrose }\end{array}$ & $\begin{array}{l}\text { Plastische } \\
\text { Chirurgie }\end{array}$ & $2020-2021$ & 14522 \\
\hline $\begin{array}{l}\text { LMU } \\
\text { München }\end{array}$ & Friedrich-Baur-Stiftung & $\begin{array}{l}\text { Induktion der Lymphangiogenese in klinisch } \\
\text { verwendetem Hautersatzmaterial, durch Kokultur } \\
\text { von lymphatischen Endothelzellen (LECs) und } \\
\text { Adipose Tissue Derived Stem Cells (ASCs) unter } \\
\text { hypoxischen Bedingungen }\end{array}$ & $\begin{array}{l}\text { Plastische } \\
\text { Chirurgie }\end{array}$ & $2019-2020$ & 8000 \\
\hline Regensburg & $\begin{array}{l}\text { Virtuelle Hochschule } \\
\text { Bayern - Forschergruppe }\end{array}$ & $\begin{array}{l}\text { Ambulante Notfallversorgung von Hand- } \\
\text { verletzungen }\end{array}$ & $\begin{array}{l}\text { Orthopädie, Unfall- } \\
\text { chirurgie, rekon- } \\
\text { struktive Chirurgie }\end{array}$ & seit 2016 & 44753 \\
\hline Regensburg & Virtuelle Hochschule Bayern & $\begin{array}{l}\text { 3-D-in-vivo-Tumor-Modell, Vielfältige Einsatz- } \\
\text { möglichkeiten des 3D-in-vivo-Tumor-Modells } \\
\text { in der Forschung sowie in der Ausbildung von } \\
\text { Studierenden der Medizin }\end{array}$ & $\begin{array}{l}\text { Orthopädie, Unfall- } \\
\text { chirurgie, rekon- } \\
\text { struktive Chirurgie }\end{array}$ & 2020 & 55000 \\
\hline Regensburg & Virtuelle Hochschule Bayern & $\begin{array}{l}\text { Angewandte klinische Anatomie und biologische } \\
\text { Rekonstruktionen - Schwerpunkt auf Sarkome der } \\
\text { unteren Extremität }\end{array}$ & $\begin{array}{l}\text { Orthopädie, Unfall- } \\
\text { chirurgie, rekon- } \\
\text { struktive Chirurgie }\end{array}$ & 2020 & 55000 \\
\hline Regensburg & $\begin{array}{l}\text { Manfred Roth Stiftung - } \\
\text { Forschergruppe }\end{array}$ & $\begin{array}{l}\text { Untersuchung physiologischer und physika- } \\
\text { lischer Auswirkungen der Vakuum-Therapie auf } \\
\text { Weichteilgewebe zum Verständnis der zu Grunde } \\
\text { liegenden Wirkmechanismen }\end{array}$ & $\begin{array}{l}\text { Orthopädie, Unfall- } \\
\text { chirurgie, rekon- } \\
\text { struktive Chirurgie }\end{array}$ & seit 2017 & 30000 \\
\hline Regensburg & $\begin{array}{l}\text { Virtuelle Hochschule } \\
\text { Bayern - Forschergruppe }\end{array}$ & $\begin{array}{l}\text { Rekonstruktive Verfahren zur Wiederherstellung } \\
\text { der weiblichen Brust }\end{array}$ & $\begin{array}{l}\text { Orthopädie, Unfall- } \\
\text { chirurgie, rekon- } \\
\text { struktive Chirurgie }\end{array}$ & seit 2018 & 69942 \\
\hline Regensburg & $\begin{array}{l}\text { Else Kröner-Fresenius-Stif- } \\
\text { tung - Forschergruppe }\end{array}$ & $\begin{array}{l}\text { Aufbau eines Cancer Comprehensive Center (CCC) } \\
\text { Upper Myanmar (mit Schwerpunkt Tumorortho- } \\
\text { pädie und Rekonstruktionschirurgie) }\end{array}$ & $\begin{array}{l}\text { Orthopädie, Unfall- } \\
\text { chirurgie, rekon- } \\
\text { struktive Chirurgie }\end{array}$ & seit 2020 & 583060 \\
\hline Regensburg & Bundeswehr - Forschergruppe & $\begin{array}{l}\text { Forschungsvorhaben „Optimierung der Trans- } \\
\text { plantatkonservierung durch unidirektionale } \\
\text { extrakorporale Perfusion von Myokutanlappen im } \\
\text { Großtier-Replantations-Modell (Schwein) }\end{array}$ & $\begin{array}{l}\text { Orthopädie, Unfall- } \\
\text { chirurgie, rekon- } \\
\text { struktive Chirurgie }\end{array}$ & seit 2020 & 281820 \\
\hline Regensburg & $\begin{array}{l}\text { Helmholtz-Gesellschaft Berlin/ } \\
\text { Teltow - Forschergruppe }\end{array}$ & HZGArc-8 & $\begin{array}{l}\text { Orthopädie, Unfall- } \\
\text { chirurgie, rekon- } \\
\text { struktive Chirurgie }\end{array}$ & seit 2020 & 1010000 \\
\hline TU München & $\begin{array}{l}\text { Industrie - Forschergruppe } \\
\text { (BIOTRONIK SE \& Co. KG }\end{array}$ & CAE-Herzmodellerstellung & Medizin Allgemein & $2020-2021$ & 190500 \\
\hline Tübingen & $\begin{array}{l}\text { AKF - Angewandte Klinische For- } \\
\text { schung - intramurale Förderung }\end{array}$ & PhantomAR & $\begin{array}{l}\text { Plastische } \\
\text { Chirurgie }\end{array}$ & 2021 & 90000 \\
\hline Tübingen & Lorenz Böhler Fonds & $\begin{array}{l}\text { Therapeutische Effekte extrazellulärer Vesikel } \\
\text { auf die Regeneration des Nervus medianus nach }\end{array}$ & $\begin{array}{l}\text { Plastische } \\
\text { Chirurgie }\end{array}$ & 2020 & 37000 \\
\hline
\end{tabular}


von chirurgischen Forschungsanträgen im letzten Jahrzehnt - bei gleichbleibendem Finanzierungsvolumen - erheblich zurückgegangen ist [8]. Dies ist auch auf eine Verringerung der chirurgischen Forschungsaktivitäten insgesamt zurückzuführen [9-11].

Erfreulicherweise zeigt sich in den vergangenen Jahren und im nun dritten Forschungsförderungsbericht der universitären Plastischen Chirurgie in Deutschland weiterhin ein positiver Trend, was das Einwerben von Forschungsmitteln großer öffentlicher, nationaler Förderinstitute angeht. Während die Anzahl bewilligter DFG-Forschungsanträge 2015/2016 bereits 21 Anträge betrug und 2017/18 mit 19 Anträgen nahezu stabil blieb, konnten $2019 / 20$ sogar 24 bewilligte DFG-Anträge identifiziert werden. Auch die Anzahl der insgesamt bewilligten Anträge von DFG, BMBF, BMWi, BMG und EU konnte von 23 (2015/16) über 27 (2017/18) auf 31 (2019/20) Anträge gesteigert werden. Weiterhin sollte hervorgehoben werden, dass die bewilligten DFG-Anträge in 50\% der Fälle dem Fachkollegium 205-27 Orthopädie, Unfallchirurgie, rekonstruktive Chirurgie zugeordnet waren, während 2017/18 lediglich $22 \%$ der bewilligten DFG-Anträge dem für unser Fachgebiet vorgesehenem Fachkollegium Unfallchirurgie und Orthopädie zugeordnet waren.

Auch die Forschungsleistung insgesamt zeigt eine positive Tendenz in der Plastischen Chirurgie. Während 2017/18 noch eine Gesamtzahl von 69 Anträgen bei öffentlichen, nicht-öffentlichen, industriellen oder anderen Förderinstituten mitgeteilt wurden, erhöhte sich die Zahl 2019/20 auf 93 gestellte Anträge. Dies ist insbesondere vor dem Hintergrund zurückgehender Antragszahlen in den anderen chirurgischen Fachgebieten von großer Bedeutung [8-11].

Die hohe Zahl der bewilligten Anträge bei öffentlichen Förderinstituten, mit $>70 \%$ aller Forschungsanträge, zeigt die ausgezeichnete Qualität der Anträge aus der Plastischen Chirurgie und sollte dazu ermutigen, sich weiterhin in dieser Hinsicht zu engagieren. Vergleichsweise liegt die Zahl der Bewilligungen in anderen Fachgebieten zwischen 30 und $40 \%$ aller Förderanträge [12].

In verschiedenen kürzlich publizierten Studien konnte nachgewiesen werden, dass die Organisationsstruktur der Plastischen Chirurgie an den universitären Einrichtungen wesentlichen Einfluss auf die Publikationsleistung und -qualität, die vorhandenen Ressourcen und die Forschungsinfrastruktur hat $[2-4,13,14]$. Nachdem in der hier vorgelegten Arbeit über $84 \%$ der bewilligten DFG-Anträge von Kliniken für Plastische Chirurgie stammten, während lediglich je $8 \%$ von Abteilungen und Sektionen für Plastische Chirurgie ausgingen, unterstreichen diese Daten, dass ein direkter Zusammenhang zwischen dem Grad der Eigenständigkeit und der Forschungsleistung besteht.

Die hier vorgestellte Zusammenstellung der Förderanträge ist nicht ohne Limitationen. Eine Limitierung basiert auf dem Fragebogen-Design der Studie. Neben den öffentlich zugänglichen Informationen über das GEPRIS-Portal basierten die Daten auf den im Vertrauen erhobenen Angaben der Befragten der universitären Einrichtungen. Dies ist insbesondere im Hinblick auf abgelehnte oder beantragte Fördermittel zu berücksichtigen. Während bewilligte Drittmittel wahrscheinlich zu einem großen Anteil mitgeteilt wurden, könnten abgelehnte oder beantragte Anträge „under-reported“ sein. Aus diesem Grund sollte auch die kalkulierte Förderquote mit Vorsicht interpretiert werden, da die wirkliche Anzahl abgelehnter Anträge höher als berichtet sein könnte.

\section{Schlussfolgerung}

Mithilfe der vorgelegten Forschungsförderungsberichte des Konvents der leitenden universitär tätigen Plastischen Chirurgen der DGPRÄC erfolgt die Veröffentlichung der exzellenten gemeinschaftlichen akademischen Forschung in unserem Fachgebiet. Nachdem die Plastische Chirurgie weiterhin in den großen öffentlichen Förderinstituten nicht als eigenständiges Fachgebiet abgebildet wird, ist nur so eine transparente Abbildung der plastischchirurgischen Forschungsaktivität möglich. Nur das starke Auftreten als Gemeinschaft Plastischer Chirurgen verbessert die Chancen darauf, langfristig mehr Gewicht in den Fördereinrichtungen zu erlangen.

Interessenskonflikt

Die Autoren geben an, dass kein Interessenskonflikt besteht.

\section{Literatur}

[1] Larson KE, Gastman B. Sources of federal funding in plastic and reconstructive surgery research. Plast Reconstr Surg 2014; 133: 1289-1294. doi:10.1097/PRS.0000000000000083

[2] Schubert CD, Haertnagl F, Elsner ] et al. [Are autonomous departments better research institutions? Influence of independence on publication performance and study-types in academic Plastic Surgery]. Sind autonome Kliniken die besseren Forschungsstandorte? Einfluss von Eigenständigkeit auf Publikationsleistung und Studientypen in der akademischen Plastischen Chirurgie. Handchir Mikrochir Plast Chir 2018; 50: 277-283. doi:10.1055/a-0645-7007

[3] Liu P, Singh M, Eriksson E. Academic Status of Plastic Surgery in the United States and the Relevance of Independence. Handchir Mikrochir Plast Chir 2016; 48: 65-68. doi:10.1055/s-0042-104373

[4] Giunta RE, Machens HG. [Science and research in academic plastic surgery in Germany]. Zur aktuellen Situation von Wissenschaft und Forschung der Plastischen Chirurgie in Deutschland. Handchir Mikrochir Plast Chir 2009; 41: 359-363. doi:10.1055/s-0029-1225365

[5] Giunta RE, Mollhoff N, Horch RE et al. [Registry Research Funding of the German Society of Plastic, Reconstructive and Aesthetic Surgeons (DGPRAC) and Research Funding Report 2017/2018]. Register Forschungsförderung der Deutschen Gesellschaft der Plastischen, Rekonstruktiven und Ästhetischen Chirurgen (DGPRÄC) und Forschungsförderungs-Bericht 2017/2018. Handchir Mikrochir Plast Chir 2018; 50: 414-421. doi:10.1055/a-0770-3485

[6] Giunta RE, Horch RE, Prantl L et al. [Registry Research Funding of the German Society of Plastic, Reconstructive and Aesthetic Surgeons (DGPRAC) and Research Funding Report 2015/2016]. Register Forschungsförderung der Deutschen Gesellschaft der Plastischen, Rekonstruktiven und Ästhetischen Chirurgen (DGPRÄC) und Forschungsförderungs-Bericht 2015/2016. Handchir Mikrochir Plast Chir 2016; 48: 370-373. doi:10.1055/s-0042-121417

[7] GEPRIS - Geförderte Projekte der Deutschen Forschungsgemeinschaft. Im Internet: http://gepris.dfg.de; Stand: 07.08.2020

[8] Narahari AK, Mehaffey JH, Hawkins RB et al. Surgeon Scientists Are Disproportionately Affected by Declining NIH Funding Rates. Journal of the American College of Surgeons 2018; 226: 474-481. doi:10.1016/j. jamcollsurg.2017.12.047

[9] Keswani SG, Moles CM, Morowitz M et al. The Future of Basic Science in Academic Surgery: Identifying Barriers to Success for 
Surgeon-scientists. Ann Surg 2017; 265: 1053-1059. doi:10.1097| SLA.0000000000002009

[10] [Anonym]. More surgeons must start doing basic science. Nature 2017; 544: 393-394. doi:10.1038/544393b

[11] Besner GE. Patterns of National Institutes of Health Grant Funding to Surgical Research and Scholarly Productivity in the United States. Ann Surg 2020. doi:10.1097/SLA.0000000000004187

[12] Bearbeitungsdauer und Erfolgsquoten - DFG. Im Internet: https:// www.dfg.de/dfg_profil/zahlen_fakten/statistik/bearbeitungsdauer/ index.html\#anker33351144; Stand: 07.08.2020

[13] Ehrl D, Giunta R. [Interdisciplinary Plastic Surgery - Chances, Conditions and Barriers of Reconstructive Microsurgery in Maximum Care Hospitals]. Interdisziplinäre Plastische Chirurgie - Chancen, Voraussetzungen und Hemmnisse der Rekonstruktiven Mikrochirurgie an Kliniken der Maximalversorgung. Handchir Mikrochir Plast Chir 2019; 51: 284-294. doi:10.1055/a-0898-3669

[14] Schubert CD, Leitsch S, Haertnagl F et al. [Independence in Plastic Surgery - Benefit or Barrier? Analysis of the Publication Performance in Academic Plastic Surgery Depending on Varying Organisational Structures]. Vorteil durch Eigenständigkeit? Analyse der Publikationsleistung der universitären Plastischen Chirurgie in verschiedenen Organisationsstrukturen. Handchir Mikrochir Plast Chir 2015; 47: 213-221. doi:10.1055/s-0035-1555954 